\title{
Teorías subjetivas en profesores y su formación profesional*
}

\author{
DAVID JORGE CUADRA MARTÍNEZ \\ Universidad Santo Tomás, Copiapó, Chile \\ JORGE RENÉ CATALÁN AHUMADA \\ Universidad de La Serena, La Serena, Chile
}

\section{RESUMEN}

El artículo presenta un estudio descriptivo interpretativo de teorías subjetivas sobre profesores y el proceso de formación docente, y su relación con las prácticas educativas. Haciendo uso de metodología cualitativa, se aplicaron entrevistas con un grupo de discusión formado por doce profesores(ras) de una escuela municipal, de una comuna de la región de Atacama, Chile. Se explica la formación docente sobre todo mediante teorías subjetivas que consideran experiencias biográficas ocurridas antes, durante y posteriormente a la formación inicial, como fuentes importantes de aprendizaje de la profesión, y que desvalorizan el impacto del aprendizaje de conocimientos semánticos. Destaca una teoría subjetiva que orienta la acción formativa del sentido subjetivo otorgado a la formación, profesión y enseñanza. En la discusión, se analiza el impacto de tales teorías subjetivas en la formación docente, considerando su contenido, estructura y capacidad explicativa.

PALABRAS CLAVE

formación docente; saber pedagógico; subjetividad; educación básica; profesión docente.

* Este trabajo presenta los principales resultados obtenidos en la tesis de maestría en psicología con mención en psicología educacional, Universidad de La Serena, Chile. 


\title{
SUBJECTIVE THEORIES ON PROFESSORS IN THEIR PROFESSIONAL TRAINING
}

\begin{abstract}
This article presents a descriptive interpretive study of teachers' subjective theories that they bring to the teacher education process and of their relationship with educational practices. Using a qualitative methodology, interviews were applied and an in depth group discussion for twelve teachers of a basic municipal school, of a community in the Atacama's region, Chile. The teachers' education is explained principally using subjective theories that consider biographical experiences that occurred before, during, and later in the initial formation, as important sources of learning for the profession and that devalue the impact of the learning of semantic knowledge. Highlighted is a subjective theory that orientates the formative action from the subjective sense given to the formation, profession and teaching. In the discussion the impact of these subjective theories on the teachers' education is analyzed, considering the content, structure and explanatory capacity.

\section{KEYWORDS}

teacher education; to know pedagogically; subjectivity; basic education; teaching profession.
\end{abstract}

\section{TEORIAS SUBJETIVAS SOBRE PROFESSORES E SUA FORMAÇÃO PROFISSIONAL}

\section{RESUMO}

O texto apresenta um estudo descritivo interpretativo de teorias subjetivas sobre professores e o processo de formação docente e sua relação com as práticas educativas. Usando a metodologia qualitativa, foram aplicadas entrevistas com um grupo de discussão composto de doze professores(as) de uma escola municipal em uma área da região do Atacama, Chile. A formação de professores é explicada principalmente por teorias subjetivas que consideram experiências biográficas ocorridas antes, durante e após a formação inicial como fontes importantes de aprendizagem da profissão, desvalorizando o impacto da aprendizagem do conhecimento semântico. $\mathrm{O}$ artigo destaca uma teoria subjetiva que orienta a ação de formação do sentido subjetivo dado à formação, profissão e ensino. Na discussão, analisa o impacto dessas teorias subjetivas na formação de professores, tendo em conta o conteúdo, a estrutura e a capacidade explicativa delas.

PALAVRAS-CHAVE

formação de professores; conhecimento pedagógico; subjetividade; educação básica; profissão docente. 


\section{INTRODUCCIÓN}

En las últimas décadas, se ha considerado el profesor una variable clave en el mejoramiento de la educación, sin que exista política o programa de intervención que no lo involucre en algún punto (Fullan, 2002; Vaillant, 2005). De hecho, en Chile, la reforma de la educación incorpora el fortalecimiento de la formación docente como uno de sus cuatro ámbitos de intervención (Ministerio de Educación, 2004).

No obstante lo anterior, la relación entre el proceso formativo, la praxis educativa y los resultados no son del todo claros. Así, Vezub (2007) explica cómo la formación inicial de profesores parece ejercer bajo impacto sobre las instituciones escolares, puesto que los docentes recién egresados terminan adaptándose rápidamente a las rutinas escolares. También se reconoce la baja relación entre los programas de capacitación tradicional y la transferencia de lo aprendido a las prácticas pedagógicas.

Dado tales resultados, los métodos actuales para la formación docente apuntan a rescatar los aprendizajes identificados por los mismos profesores como generadores de prácticas educativas exitosas (Raczynski; Muñoz,2007). Lo anterior es factible de abordar desde el paradigma de investigación del pensamiento del profesor, que permite asumir que éstos son profesionales que actúan racionalmente, toman decisiones, y la mayoría de sus acciones tienen algún tipo de relación con sus pensamientos. Este estudio se enmarca dentro de este modelo de investigación, específicamente en la corriente de investigación de las creencias y teorías subjetivas del profesor (Catalán, 2011; Clark; Peterson, 1990; Marcelo, 1987, 2013; Shulman, 1986).

Así, el problema de estudio se aborda desde las teorías subjetivas, definidas como interpretaciones e hipótesis con carácter argumentativo, acerca de sí mismos y su contexto (Catalán, 2004; Flick, 2004). Interesa aquí conocer las explicaciones de los propios involucrados acerca de su formación profesional y la forma cómo la relacionan con las prácticas educativas.

Com esta reflexión surge el siguiente problema de estudio: ¿Cuáles son las teorías subjetivas de los profesores de una escuela de primaria municipal en una comuna de la región de Atacama acerca del proceso de formación docente y de su relación con las prácticas educativas? El objetivo general del estudio que se reporta ha sido describir e interpretar estas teorías subjetivas, y en concreto:

1) Describir las experiencias que los profesores relacionan con su formación docente.

2) Identificar sus explicaciones acerca de los efectos de la formación inicial en la práctica educativa.

3) Determinar sus explicaciones de los efectos de la formación continua en la práctica educativa.

4) Identificar sus explicaciones de los efectos de las experiencias laborales formativas en la práctica educativa.

5) Determinar las hipótesis que presentan acerca de la formación docente y las prácticas educativas que consideran exitosas y no exitosas. 
En cuanto a la pertinencia y relevancia de este estudio, hay que señalar que los hallazgos que aquí se exponen contribuyen a una mayor comprensión del proceso formativo del docente, dada la urgente necesidad de lograr mejoras en tal proceso (Manzi; González; Sun, 2011; Marcelo, 2013; Vezub, 2007). Eso rescatando el propio marco interpretativo del profesor desde un tipo particular de creencias, o sea, sus teorías subjetivas, dada la influencia decisiva que éstas ejercen sobre el aprendizaje e implementación de las innovaciones educativas (Marcelo, 2013), lo que además permite arrojar luces sobre sus procesos reflexivos, ambos factores señalados como clave para el desarrollo profesional docente.

\section{MARCO TEÓRICO}

\section{FORMACIÓN DOCENTE}

La definición que se tenga de formación docente dependerá del modelo de formación que se encuentre en su base. Marcelo (1989) la define como un campo de investigación científico teórico-práctico que estudia el proceso mediante el que los profesores se integran en experiencias de aprendizaje que les permiten una mejora de sus conocimientos, destrezas y disposiciones, permitiéndoles, así, desarrollar intervenciones educativas profesionales. De Lella (1999), por otra parte, define formación docente como un proceso de permanente adquisición, estructuración y reestructuración de conocimientos, habilidades y valores para el desempeño de la función docente; a su vez, puede dividirse en inicial y continua. Rodríguez (1994) advierte una doble función formativa para el profesor: por una parte, la que corresponde a los conocimientos de enseñanza, y por otra, a las habilidades de desarrollar la pedagogía, que además de considerar las formas de enseñanza, incluyen gestión educativa, planificación y evaluación del currículum, planificación y evaluación institucional.

Torres (1998) incorpora el concepto de aprendizaje permanente, considerando que la formación del profesor no sólo es el resultado de los aprendizajes académicos, sino que de aquellos ocurridos durante toda la vida, dentro y fuera de la escuela.

En síntesis, se puede definir formación docente como el proceso mediante el cual el profesor se va formando como un profesional de la educación por medio de una serie de experiencias formales e informales de aprendizaje, ocurridas durante toda la vida.

Tal proceso formativo dependerá de la posición epistemológica asumida para entender qué es currículum, enseñanza, educación, escuela y profesión docente. Davini (1995) propone los siguientes modelos: práctico artesanal, academicista, tecnicista eficientista y hermenéutico reflexivo. Desde este último, se promueve la profesionalización docente y se pone acento en los procesos reflexivos sobre la propia práctica para llevar a cabo la labor de enseñanza.

Otros autores resumen el proceso a sólo dos modelos de formación docente. Moral (2000) plantea que en la formación académica-tecnológica se considera al docente como un técnico, mientras que en el modelo práctico, personalista y 
reconstruccionista social, se le atribuye el estatus de profesional. Este último se sustenta en el paradigma del pensamiento del profesor e identifica al docente como un profesional reflexivo.

\section{EL PARADIGMA DE INVESTIGACIÓN CIENTÍFICA DEL PENSAMIENTO DEL PROFESOR}

Durante la década de 1970 se inicia un proceso de reconceptualización de la educación, que desplaza el estudio de la conducta y la formación centrada en competencias técnicas y lo substituye por el del pensamiento del profesor. Se asume que lo que los profesores creen, piensan o conocen se encuentra relacionado con sus prácticas educativas, la significación otorgada al currículum, las relaciones profesor-alumno y la actitud frente a las innovaciones educativas (Martínez et al., 2001). Clark y Peterson (1990) proponen una clasificación en tres grandes dominios que agrupan los diversos enfoques de investigación del pensamiento del profesor: la planificación docente, los pensamientos y decisiones interactivas y las teorías y creencias. Se consideran las teorías subjetivas como una corriente de investigación dentro del último dominio propuesto por estos autores.

Catalán (2011) establece una asociación entre el pensamiento del profesor y su conocimiento profesional: el primero serviría de sustento para el desarrollo del segundo, posicionando como base central del proceso de cambio al acto reflexivo. Esto último se considera fundamental para el desarrollo profesional, y desde este planteamiento, se consideran las teorías subjetivas como tipo de conocimiento el foco central de la reflexión.

\section{TEORÍAS SUBJETIVAS}

Se definen las teorías subjetivas como construcciones personales explicativas implícitas o explícitas, con una estructura lingüística argumentativa y del tipo causaefecto, que permiten interpretar el mundo así como el propio comportamiento; son de carácter individual y se vinculan con lo social tanto en su comunicación como en su formación (Catalán, 1997; Flick, 2004). Para Groeben y Scheele (apud Arístegui et al., 2004), cumplen una función central en la persona, visto que permiten explicar y predecir, a la vez que orientan el comportamiento, guían la acción y mejoran la autovaloración.

Grotjahn (1991), al caracterizar las teorías subjetivas, plantea que serían de carácter individual, estables y de duración prolongada; se relacionan con la propia persona y su mundo, poseen una compleja estructura argumentativa, cumplen una función paralela a la de las teorías científicas en el sentido que posibilitan explicar y predecir fenómenos y pueden explicitarse mediante el diálogo. Los supuestos básicos de este enfoque de investigación son (Flick, 2004):

a) En su vida cotidiana, la gente desarrolla teorías sobre el funcionamiento del mundo y sus actividades.

b) Las teorías desarrolladas por la gente se aplican y evalúan en las actividades del diario vivir.

c) La estructura lingüística de estas teorías se asemeja a la de las teorías científicas, y sus argumentos se organizan de forma interdependiente. 


\section{TEORÍAS SUBJETIVAS Y FORMACIÓN DOCENTE}

Fox (1983 apud Jiménez; Feliciano, 2006), en su estudio sobre teorías implícitas en docentes universitarios, encontró dos tipos de teorías acerca del aprendizaje y lel rol del profesor: la primera de ellas se denomina teorías simples, en cuya estructura lingüística se denota una relación limitada entre enseñanza y aprendizaje, y en la que, además, el docente asume un rol de formador directivo; la segunda de ellas, teorías desarrolladas, presenta un enunciado que considera al proceso de enseñanza $\mathrm{y}$ aprendizaje complejo, en el que el estudiante es quien asume un rol activo.

Otros autores también han subrayado la simplicidad de las teorías subjetivas de docentes. Un estudio realizado por Catalán (2004) sobre teorías subjetivas de la formación inicial de educadoras de párvulos en formadores y formadoras, advierte sobre una tendencia a la escasez de las mismas y a la presencia de juicios descriptivos sobre el tema abordado, por lo que se puede interpretar que el grupo de docentes estudiados presentaría un nivel preteórico en la significación de la formación inicial de educadoras de párvulos. Cuadra (2009) ha encontrado hallazgos similares a los referidos por Catalán (2004) respecto al bajo estatus teórico de las teorías subjetivas de docentes, y a los de Fox (1983 apud Jiménez; Feliciano, 2006), haciendo referencia a la simplicidad de las explicaciones que profesores de educación básica de una escuela de bajo desempeño presentan sobre enseñanza y aprendizaje en alumnos(nas).

Castro (2008) realiza un estudio sobre el cambio de teorías subjetivas de profesores respecto a la enseñanza y aprendizaje de valores. A modo general, se observa que resulta posible promover cambios en las teorías subjetivas de docentes por medio de su participación en una capacitación de educación en valores, que se pueden resumir en el mayor autonomía, protagonismo y percepción de eficacia respecto de la enseñanza de valores (Castro; Cárcamo, 2012).

\section{MÉTODO}

\section{TIPO DE ESTUDIO Y DISEÑO}

Es una investigación de tipo descriptiva interpretativa, en la que se utilizó metodología cualitativa con el fin de caracterizar al objeto de estudio (Krause, 1995). Se define como un método inductivo, que considera al sujeto de estudio y su contexto de manera holística, asume los efectos del propio investigador sobre los sujetos estudiados, interactúa con éstos de la manera más natural posible y comprende el fenómeno de estudio desde el marco de interpretación de los propios actores (Taylor; Bogdan, 1987).

\section{SUJETOS}

Se trabajó con 12 profesores(ras) de una escuela básica municipal, de una comuna de la región de Atacama, Chile. Al momento del estudio la escuela contaba con 390 alumnos(nas) matriculados, distribuidos en los cursos de prekinder a octavo año de primaria. Como criterios de selección de los participantes se estableció: 
a) Que al momento de la entrevista tuvieran el título profesional de profesor.

b) Que su función en la escuela fuera o hubiese sido la docencia.

c) Que presentaran como mínimo dos años de experiencia profesional como docentes.

Cuadro 1 - Caracterización de los sujetos de investigación

\begin{tabular}{|c|c|c|c|c|c|c|c|c|c|c|c|c|c|c|c|}
\hline & \multicolumn{2}{|c|}{ Sexo } & \multicolumn{3}{|c|}{ Rango edad en años } & \multicolumn{3}{|c|}{$\begin{array}{c}\text { Formación } \\
\text { inicial }\end{array}$} & \multicolumn{5}{|c|}{ Formación continua } & \multicolumn{2}{|c|}{$\begin{array}{c}\text { Situación } \\
\text { contractual }\end{array}$} \\
\hline & $\mathrm{H}$ & M & 40 a 50 & 51 a 60 & 60 o más & 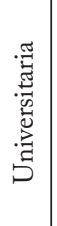 & 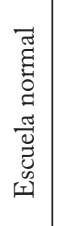 & 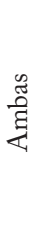 & 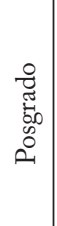 & $\begin{array}{l}0 \\
\text { 营 } \\
\text { م }\end{array}$ & 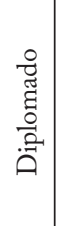 & 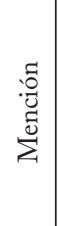 & 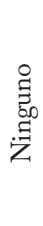 & 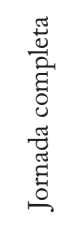 & 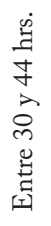 \\
\hline Número & 7 & 5 & 3 & 7 & 2 & 5 & 3 & 4 & 1 & 9 & 0 & 2 & 1 & 7 & 5 \\
\hline
\end{tabular}

Fuente: Banco de dados de la encuesta.

Elaborado por los autores.

\section{PROCEDIMIENTO DE RECOLECCIÓN DE DATOS}

Se contactó inicialmente a la dirección de la escuela solicitándole colaboración para la realización del estudio. Se explicó el objetivo, así como la metodología de la investigación, y se invitó a los docentes a participar, informándoles la finalidad del estudio: "conocer la forma cómo ellos caracterizan la formación docente". Para lograr mayor credibilidad en el estudio, se utilizaron dos instrumentos de recolección de información a modo de triangulación de métodos:

\section{Entrevista en profundidad}

Método de recolección de información que se basa en una conversación sistemática, en la que el investigador ayuda al sujeto investigado a reproducir la realidad que ha construido (Ruiz, 2003). Se consideró la proposición de Flick (2004) respecto a la indagación de episodios biográficos, dado que precisamente son éstos los que actúan como fuentes de conocimiento para la construcción de teorías subjetivas. Así, se buscó la producción de tres tipos de información en los sujetos:

a) Episodios específicos informados espontáneamente.

b) Episodios repetidos informados espontáneamente.

c) Definiciones subjetivas de los tópicos abordados.

Se realizaron 6 entrevistas en profundidad en instalaciones de la escuela, empleándose para ello una guía temática que consideró:

a) La forma como caracteriza la formación inicial. 
b) La formación continua.

c) Las experiencias laborales formativas.

d) La formación docente actual.

e) El modo cómo explica su impacto en la educación.

\section{Grupo de discusión}

Técnica de investigación social que trabaja con producciones lingüísticas generadas por un grupo de personas en un ambiente lo más natural posible.

Participaron 6 profesores que no se sometieron a la entrevista en profundidad. Se usó la misma guía temática y la proposición de Flick (2004) utilizada en la entrevista en profundidad.

Ambos instrumentos se aplicaron en instalaciones de la escuela. Los datos obtenidos fueron registrados en forma audiovisual y posteriormente transcritos.

\section{PLAN DE ANÁLISIS DE DATOS}

Se realizó el análisis de los datos mediante la proposición de análisis de contenido de grounded theory. Se trabajó con tres tipos de codificaciones (Strauss; Corbin, 2002):

a) Codificación abierta: se redujo el corpus a categorías más generales, empleándose para ello el método comparativo constante. En esta etapa se reconocieron teorías subjetivas en el texto, tomando por base la proposición de Catalán (2010), identificándose hipótesis formuladas expresamente o inferidas por el investigador.

b) Codificación axial: se construyeron cuatro ejes de análisis (categorías) que agruparon al conjunto de códigos, empleándose para ello el método comparativo constante.

c) Codificación selectiva: en esta etapa de análisis se asignó una de las categorías como núcleo o dimensión central de los hallazgos, estableciéndose al mismo tiempo relaciones entre ellas que permitieron comprender el fenómeno de estudio.

En forma complementar, se aplicaron análisis específicos para las teorías subjetivas (Catalán, 2005, 2010). Se hizo referencia al estatus teórico de las teorías subjetivas, su estructura y la orientación a la acción.

\section{HALLAZGOS}

\section{PRIMEROS PASOS EN LA CONSTRUCCIÓN DEL SIGNIFICADO DE LA PROFESIÓN DOCENTE: LA FORMACIÓN PRECOZ DE LA PROFESIÓN}

Teorías subjetivas sobre la vocación del profesor

Se observan teorías subjetivas que en su estructura argumentativa determinan experiencias biográficas de la infancia como causa de la vocación y elección de la 
carrera profesional. Los docentes explican que su profesor(ora) de enseñanza primaria había utilizado con ellos prácticas pedagógicas y/o una relación interpersonal que les causara admiración, que les pareciera modelar, afectuosa o comprometida, lo que les habría generado la motivación para ser docente y el modelaje de esas prácticas, conocimiento que aplican a días de hoy en algún nivel con sus propios alumnos. La vocación para cursar pedagogía comienza a configurarse también por influencia de un familiar que ha sido significativo para el profesor durante su infancia y/o adolescencia.

Un segundo factor que los docentes señalan como influjo en su decisión de estudiar pedagogía lo representa la posibilidad de acceso a educación superior. Explican que cursar pedagogía representó la posibilidad de lograr una carrera profesional que les otorgara ascenso social y económico, de manera rápida y con posibilidad de gratuidad en los estudios.

Grupo de discusión: ${ }^{1}$

- Profesora 4: ... me entiende, porque usted sabe que un profesor ganamos poco, pero me gusta, tengo vocación y creo que busco las alternativas para llegar, y creo que la elegí por eso, porque ese profesor me marcó.

- Profesor 5: ... varios familiares son profesores, entonces era como un patrón que uno tiene...

- Profesor 3: ... entonces en ese tiempo, por a lo mejor problemas familiares o por condición familiar, había que buscar alternativas de seguir estudiando, recursos económicos más que nada, entonces, una de las cosas la escuela normal, sonaba mucho, habían becas...

\section{Teorías subjetivas sobre el aprendizaje de prácticas pedagógicas}

Los profesores identifican experiencias de aprendizaje de la profesión anteriores a su formación inicial. Explican que adquirieron conocimiento profesional, siendo alumnos(nas) de enseñanza primaria, por medio de una serie de experiencias biográficas vividas en la escuela y aprendidas de la conducta de su profesor. Destacan aquí dos tipos de aprendizaje:

a) Prácticas pedagógicas que regulan la convivencia escolar, el desarrollo moral y emocional del alumno: se señala el aprendizaje del respeto, afecto y compromiso con el alumno, así como su formación en valores.

b) Prácticas pedagógicas académicas: consistentes en el aprendizaje de procedimientos sobre cómo enseñar.

1 La transcripción se mantuvo como el discurso de cada participante. 
- Profesora 2: Sí, sí, yo creo que primero así, la cercanía que ella tenía con nosotras, porque ella era una profesora muy de piel... era una profesora muy interesada en los sentires de cada uno, tenía... ella tenía demasiado respeto por lo que era cada uno de nosotros, yo creo que esa práctica yo la tengo de ella.

\section{EL APRENDIZAJE DE LA PROFESIÓN DOCENTE DURANTE LA FORMACIÓN INICIAL}

La formación inicial del profesor se caracteriza de manera diferente, según se haya cursado la escuela normal ${ }^{2}$ o la universidad. Aunque en todos los casos se señala que ese paso formativo sólo representa un porcentaje mínimo del conocimiento profesional y que se presenta una mayor calidad en la educación normalista respecto la universitaria. Esto último se argumenta mediante la siguiente teoría subjetiva latente: existen políticas educativas gubernamentales de baja exigencia y poco control para la formación docente actual, así que los centros de enseñanza de esa profesión tienen un sentido económico de la educación que facilita el acceso a est carrera y disminuye la calidad de la enseñanza, y el resultado son profesores menos competentes y comprometidos.

- Profesor 6: ahora, por ejemplo, hay... se puede adquirir la profesión en un instituto de formación que no tiene nivel universitario, hay algunos que estudian por correspondencia, que no es lo mismo que presencial, que participar de un programa, depende mucho del programa que sean aprobados para la formación docente.

Un análisis más detallado manifiesta teorías subjetivas que hacen referencia a los siguientes factores que impactan la formación profesional durante ese período:

a) El currículum formal en la formación del profesor: los docentes refieren que durante el proceso formativo se imparten tres tipos de conocimientos: semántico o teórico, característico de la enseñanza universitaria, evaluado como complejo, no ajustado a la realidad escolar, y en muchos aspectos inútil. De procedimiento, impartido fundamentalmente en la escuela normal, y respecto a la universidad, se considera que su enseñanza es mínima y se adquiere sobre todo, y en algún nivel, durante la práctica profesional. Tal factor resulta ampliamente valorado, dado que implica el aprendizaje de didácticas para impartir una clase. Valorativa y ética, impartido sistemáticamente en la escuela normal; en la universidad se logra en algún nivel, aunque fundamentalmente por medio de experiencias no formales de aprendizaje.

2 Las escuelas normales chilenas fueron establecimientos del estado destinados a la formación de profesores, que operaron desde 1842 hasta el régimen del gobierno militar. Representaron un primer intento de profesionalización de la docencia, aunque su sello fue sobre todo el de una formación técnica. 
- Profesora 1: Sí, también, hartas, porque el profesor, como te digo, nos preparaba para eso, nos preparaba para ser profesor, en todo momento, la metodología, la didáctica... todo el asunto que tiene que ver con el profesor, que en la universidad se perdió.

- Profesora 2: lo que menos me ha servido son los contenidos que me pasaron, lo que menos...

b) Método de enseñanza: la totalidad de los profesores valora como método de enseñanza las experiencias de prácticas pedagógicas, fundamentalmente en centros de práctica con niveles importantes de vulnerabilidad social. Lo anterior se organiza en una teoría subjetiva, cuyo contenido manifiesta la explicación siguiente: durante la formación del profesor se aprende por experiencias prácticas de alta complejidad en escuelas vulnerables, dado que lo teórico no se ajusta a la realidad escolar.

- Profesora 1: ... nosotros en la primera parte en la escuela normal estuvimos 6 meses... después de eso, teníamos que salir a supervisar... a observar profesores que hacían sus clases, ahí teníamos práctica... ese fue el primer año, el segundo año ya teníamos que hacer práctica... entonces el profesor te mandaba a hacer la clase difícil, por ejemplo, la clase que no podía hacer... entonces tú ibas aprendiendo, ibas enseñando, ibas conociendo al alumno, ibas... ibas enseñando.

c) Características de los formadores de formadores: todos los entrevistados atribuyen características positivas al docente normalista, considerándolo un profesional de muy buen nivel y altamente comprometido con la profesión. Esa misma valoración no se atribuye al docente universitario; a este se asocia una teoría subjetiva ampliamente compartida: dado que fue formado fundamentalmente por profesionales no docentes, ha recibido una formación inicial de menor calidad respecto al normalista.

- Profesora 2: Pero sentí que la universidad en verdad no me formó como profesora, o sea, en el sentido de que no me preparó metodológicamente para poder yo transmitir la enseñanza... porque yo creo que los profesores que teníamos no estaban tampoco como preparados para formar profesores.

d) El currículum oculto durante la formación inicial: los docentes explican que durante su formación inicial, la personalidad de un profesor (o varios) y su forma de portarse había sido modelada en algún nivel. Un segundo elemento destacado por los docentes refiere a la convivencia escolar con sus pares. A ese respecto explican que episodios biográficos altamente significativos les permitieron aprender valores referidos a compañerismo, amistad, respeto y apoyo, lo que a días de hoy se pone en juego en el contexto laboral. 
- Profesor 3: ... antes, fijese, que la escuela normal estando interno, de repente nos llegaban encomiendas... entonces al que le llegaba algo lo compartía con todos, entonces ahí como que se formó un valor que nosotros tenemos, entonces e... eso también nos marcó, en el sentido de compartir las cosas...

e) Factores de la formación inicial que los docentes asocian a un desempeño exitoso o no exitoso de la profesión: el tipo de conocimiento impartido durante la educación inicial representa un factor clave en el desempeño de la profesión. A ese respecto, la enseñanza de didácticas se valora en gran medida y se adquiere fundamentalmente en las asignaturas que implican pasos prácticos y en la práctica profesional. Lo anterior se integra en teorías subjetivas que indican como causa del buen desempeño profesional el acto de aprender haciendo, y que explican la mayor o menor intensidad del uso de ese tipo de enseñanza apelando a factores sociales e institucionales. Al mismo tiempo, la totalidade de docentes desvalora la enseñanza de teorías científicas, y explican que éstas no se ajustan a la realidad escolar. En segundo lugar, se considera la enseñanza del funcionamiento de la profesión como una de las causas de la concreción de la formación profesional, dado que nutre la vocación. Implica poner a disposición del alumno información acerca del estatus social y los aspectos administrativos del funcionamiento de la profesión docente. En tercer lugar, el nivel de exigencia en la enseñanza; explican que la baja calidad en la formación del profesor se debe a un bajo nivel de exigencia, dado que a días de hoy la formación inicial tiene como meta la ganancia. Otro factor lo representa los formadores de formadores, e indican que los profesores de la carrera de pedagogía deben poseer el título de profesor para enseñar, dado que esto posibilita que los estudiantes aprendan didácticas de enseñanza, que no son de conocimiento de otras profesiones. Además, es necesario que el profesor de pedagogía lo sea al mismo tiempo de escuelas básicas o liceos, para que pueda enseñar desde esa realidad concreta.

- Profesora 1: La práctica, aprender haciendo... es poco, porque tú, tú vas trabajando, tú vas aprendiendo, vas viendo si te va a gustar o no te va a gustar lo que estás estudiando también, porque si tú te... vas a estudiar una carrera donde no te gusta, no vas a ser un buen profesional; entonces, cuándo tú vas a saber si te gusta o no te gusta, cuando saliste a trabajar, en cambio, en el camino la vamos viendo... a mí no me gustó esto, abandono.

- Profesora 2: ... lo que menos me ha servido son los contenidos que me pasaron, lo que menos...

- Profesora 3: Porque ella tenía otra formación, ella era pedagoga, ella se había preparado para formar profesores, ella estaba formada para eso, entonces la... la diferencia era notable, notable. 
f) Proposiciones de mejora para la formación inicial: Explican que la mejora de la formación inicial depende de las características de los formadores de profesores, que, en general, implica que éstos sean docentes de profesión y al mismo tiempo profesores de educación primaria; una selección de ingreso rigurosa a la carrera de pedagogía, un currículum planificado fundamentalmente con pasos prácticos que permita la adquisición de metodologías de enseñanza, y por fin, un control riguroso que involucre mayores exigencias en la formación académica en los centros de educación superior que dictan la carrera de pedagogía.

- Profesor 5: Bueno, primero, que fueran profesores destacados y básicos los que tuvieran la responsabilidad de preparar a los futuros profesores, porque ellos son los que están en el campo, te enseñan... profesores que nunca han trabajado en aula y son profesores... son profesionales y hacen clases y que sugieren cosas que son irreales, porque ellos no las han visto, no las han vivido.

\section{EL APRENDIZAJE DE LA PROFESIÓN DURANTE EL EJERCICIO PROFESIONAL}

\section{La formación continua en el aprendizaje de la profesión}

Los docentes reconocen la necesidad de mantenerse en constante formación profesional y consideran al perfeccionamiento como relevante para el adecuado ejercicio de su profesión. Esta disposición a la formación continua se explica indicando que el acelerado desarrollo del conocimiento lleva a la necesidad de que uno se actualice de forma continua en su disciplina. No obstante, se presentan teorías subjetivas que explican la dificultad para concretarlo. Tales teorías presentan en su estructura argumentativa causas de tipo administrativas (por ejemplo, permisos y horas disponibles) y de acceso a ese tipo de educación: la primera de ellas argumenta que no están dadas las condiciones administrativas para participar de espacios de formación continua presencial, por lo que se hace muy difícil concretar la formación permanente; la segunda, que la formación continua se da sobre todo a distancia, dado que en la zona de residencia no siempre se imparten cursos presenciales, por lo que el acceso a ellos es difícil.

- Profesor 4: ... qué le hace tomar cursos a distancia y no presenciales... son los tiempos, porque si yo hago curso no presencial, y tengo que contestar y mandarlo lo hago en los momentos que estoy libre; y cuando uno hace presencial, tiene que darse el tiempo de irse de su casa allá donde lo va a dictar, estar allá, esperar, hacer el curso, salir a su casa, y en qué tiempo...

Por fin, explican que la baja calidad de la formación continua se debe al sentido comercial de la enseñanza y a la mínima relación de los conocimientos impartidos con la realidad escolar. A ese respecto, tienden a cualificar los cursos de perfeccionamiento como más de lo mismo, teoría no aplicable a la realidad educativa. 
- Profesor 5: esos cursos, yo los veo, ¿sabes tú cómo los veo yo? Como un comercio, un negocio, donde no se cumple con lo que realmente uno requiere y necesita, sino que ellos están pensando más en el beneficio económico que le va a traer que lo que van a venir a entregar los colegas, si tú lo ves en todas partes están ofreciendo cursos... es puro negocio.

\section{Los contextos formativos de la profesión en el interior de la escuela}

Los profesores reconocen que el conocimiento profesional se fortalece, en gran medida, en el interior de la escuela, y un grupo más extremo indica ese tipo de aprendizaje como el más valioso de la profesión.

Explican que los factores asociados a la formación profesional en ese contexto son: la orientación que pueda ofrecer el equipo técnico de la escuela. Aunque esa posibilidad no la consideran como frecuente, y ven las reuniones técnicas como fundamentalmente administrativas; el proceso mismo de impartir clases, dado que otorga competencia, y aún más cuando la experiencia se da con variedad de alumnos(nas); el grupo de pares, es decir, los propios profesores, y en particular las relaciones interpersonales informales dado que en tales situaciones es cuando discuten sobre prácticas pedagógicas; el clima social escolar, específicamente de tipo laboral, dado que conlleva relaciones de apoyo entre pares; por fin, el reconocimiento de la labor desde cualquier fuente, puesto que permite al profesor confirmar sus prácticas pedagógicas.

- Profesora 1: Porque era leer cuestiones ahí (en los consejos técnicos), ir a leer documentos que te llegó ahora, que hace esto... no... tú en consejo técnico tienes que todos juntos planificar, donde todos en conjunto digamos este es el tipo de planificación y este es el tipo de evaluación que vamos a ir haciendo y empecemos con el niño a exigirle más.

\section{Macropolíticas de evaluación de resultados y su relación con la formación continua}

Se presenta una teoría subjetiva que relaciona las políticas de evaluación de resultados con el desarrollo de la profesión. Básicamente se explica que esos instrumentos actúan como medidas de presión que impulsan a los docentes a revisar sus prácticas pedagógicas y a esforzarse por corregirlas. El método de evaluación ${ }^{3}$ es muy criticado y rechazado, dado que se le atribuyen bajos niveles de validez y confiabilidad, y por lo mismo un alto nivel de estrés laboral, generando un fuerte

3 En Chile, la evaluación docente se implementa a partir del año 2003 como parte de las políticas de fortalecimiento de la profesión docente. Se basa en estándares que miden el desempeño de los docentes en función de criterios establecidos en el Marco para la buena enseñanza del Ministerio de Educación. Los docentes de escuelas municipales deben evaluarse cada cuatro años cuando obtienen un desempeño competente o destacado; cada dos años cuando son clasificados como básicos y deben repetir la evaluación al año siguiente cuando califican como insatisfactorio (Manzi; González; Sun, 2011). 
rechazo, por ejemplo, del sistema nacional de evaluación de resultados de aprendizaje y la evaluación docente.

- Profesor 4: ...porque si a mí me van a evaluar por lo que hago, trato de hacerlo lo mejor posible para salir bien evaluado, pero en la evaluación docente, donde le ponen una pizarra, un señor que está filmando, prepara una clase con dos meses de anticipación, la preparo, pero nunca en mi vida he preparado material en mis clases del día a día, en esa clase la preparo, pero la mando hacer, si pa eso hay profesores que preparan materiales y cobran... si es así.

\section{ORIENTACIÓN A LA ACCIÓN DEL PROFESOR DESDE LAS TEORÍAS SUBJETIVAS: EL SENTIDO DE LA FORMACIÓN, LA PROFESIÓN Y LA ENSEÑANZA}

El análisis de las teorías subjetivas del profesor permite hipotetizar la orientación a la acción que podrían llegar a tener (Catalán, 2010). Los hallazgos posibilitan clasificar tal orientación a la acción en lo que hemos denominado sentido de la formación, la profesión y la enseñanza, visto que en la base se observan teorías subjetivas inhibidoras o mantenedoras de tales posibles conductas, cuya estructura argumentativa contiene una causa que representa precisamente lo que busca el profesor, dado que la posiciona como un factor de valor.

\section{El sentido de la formación docente}

a) Sentido administrativo de la formación: quienes presentan ese sentido explican que la formación del profesor debe apuntar sobre todo al desarrollo de competencias genéricas o conductuales referidas a la responsabilidad con lo administrativo, y eso se organiza en una teoría subjetiva mantenedora de la acción, que posiciona a esos factores como causa importante del buen desempeño profesional y que podría conllevar a una orientación a la acción que consiste en la búsqueda de ese tipo formación o su establecimiento como formador.

- Profesor 4: Claro, porque a nosotros se nos formaba, bueno que nosotros distinto, incluso se nos formaba para hacer soberanía a nosotros, y la parte de la asistencia y la puntualidad eran cosas que nos marcan hasta hoy; yo venía a trabajar enfermo, y siempre llego a la hora; estoy media hora antes porque a mí me formaron distinto el profesor de ahora no cumple con las cosas.

b) Sentido didáctico de la formación: desde tal sentido los profesores explican que la formación debe apuntar al aprendizaje de didácticas de enseñanza. Critican con fuerza la formación universitaria y los cursos de formación continua, a la vez que rechazan el aprendizaje de teorías científicas. Así, la orientación a la acción del profesor se sustenta en una teoría subjetiva mantenedora de acción en la búsqueda de aprendizaje de didácticas. 
- Profesora 1: Porque tú vas... vas aprendiendo a medida que vas enseñando, aprendemos juntos, alumno y profesor vamos aprendiendo juntos, pero tú tienes detrás el profesor que te está enseñando, tú sabes que no vas a ir a meter la pata allá porque... no te sabes las materias, sino que tienes un profesor que te está enseñando, que te está diciendo esto se enseña así... usa esta metodología. Es bueno ir haciendo.

c) Sentido de sobrevivencia de la formación: se manifiesta en una teoría subjetiva mantenedora de la acción que la orienta, en algún nivel, a la búsqueda y/o participación en instancias de formación continua, pero básicamente para resistir las presiones sociales.

- Profesor 6: Como diez años, sin perfeccionarme, nada más que en perfeccionamiento, el autoperfeccionamiento que uno hace al preparar las clases, que eso es continuo no más. Empezaron a surgir algunos vacíos, especialmente con el cambio de programa... entonces fui a perfeccionarme a CPIP... entonces ahí uno se da cuenta de que el perfeccionamiento debe ser más o menos continuo, siempre está necesitando cosas.

d) Sentido económico de la formación: otorgan a la formación del profesor un sentido que la interpreta como una oportunidad que permite ganancia a las instituciones formadoras, y al profesor en formación mejorar sus ingresos económicos. Así, es posible que los docentes rechacen la formación continua, dado que constituye lucro para quienes la imparten; y a la vez, se involucren en esas instancias, pues les permite mejorar económicamente. Se infiere que en alguna medida esto inhiba la acción de perfeccionarse o la generación de altas expectativas en el perfeccionamiento.

- Profesor 6: ... e... reconozco que son como cursos para hacerlos no más, porque hay que tener ciertos cursos de perfeccionamiento, porque con el perfeccionamiento se reciben algunas bonificaciones, en fin, eso.

e) Sentido de crecimiento personal de la formación: es un sentido de la formación menos frecuente en los profesores. En su base se presenta una teoría subjetiva mantenedora de acción que en su estructura argumentativa incluye el crecimiento profesional como factor que impulsa la incorporación en instancias formativas.

- Profesora 2: Siempre me perfeccionaba porque tenía esa necesidad de aprender, de sentirme como preparado para hacer la pega.

\section{Sentido de la profesión de profesor}

a) Sentido económico: la profesión de profesor cobra sentido en la medida que permite al individuo lograr un mejor estatus social y nivel económico, ingresando en una carrera de fácil acceso. Tal explicación latente es inicia- 
dora de la acción, dado que impulsa, en algún nivel, a optar por la carrera docente en función de tales factores.

- Profesora 1: Claro, no porque una beca que me van a dar... gratis, o porque me ofrecieron que yo termino me voy a ir al extranjero y que me voy a quedar allá, porque eso también es muy común... "yo voy a estudiar pedagogía y me voy a ganar la beca y me voy a Estados Unidos y me quedo en Estados Unidos ¿Para qué me voy a quedar aquí si voy a ganar una miseria de sueldo?”

b) Sentido social de la profesión: es un sentido de la profesión menos presente en los entrevistados. En su base se observa una teoría subjetiva mantenedora de la acción que en su estructura argumentativa asocia el ejercicio de la profesión con el desarrollo de la sociedad. Quienes presentan ese sentido, se infiere que en algún nivel ejercen esforzándose para que sus alumnos contribuyan al desarrollo social.

- Grupo de discusión, profesor 4: ... entonces yo creo que la labor que nosotros tenemos dentro de la sociedad es tan importante, porque yo a veces converso con mi hermano y le digo: tú llegaste a ser doctor, pero quién te enseñó... tus primeras letras... el papel de nosotros es tan importante... a veces nosotros no nos damos cuenta la responsabilidad que tenemos frente al curso.

c) Sentido del sacrificio profesional: los docentes explican que para ser profesor se debe estar dispuesto a sacrificarse económica, familiar y psicológicamente, dado que la docencia presenta obstaculizadores y exigencias diversas, además de poco reconocimiento. De eso se infiere que la acción del docente se orienta, en algún nivel, hacia la aceptación de tales sacrificios, y por lo tanto, a la permanencia en su ejercicio profesional.

- Profesor 6: ... cuando nosotros ingresamos a la escuela Normal nos decían: ¿Sabe para qué van a estudiar?... van a estudiar una profesión la cual es bastante sacrificada, van a ganar poco, pero finalmente como que van a llegar a quererla... si tienen vocación... Entonces nos confirmaron bastante, o sea, nosotros sabíamos e... más o menos las condiciones que nos íbamos a desenvolver.

d) Sentido de la enseñanza: explican que la enseñanza tiene sentido si se logra que la escuela obtenga buenos resultados en las pruebas que miden la calidad de la educación; para otros, adquiere sentido el formar los futuros ciudadanos; por fin, se observa un sentido de enseñanza que apunta únicamente al cumplimiento administrativo, dado que los alumnos(nas) no se encuentran motivados con el aprendizaje. En cualquier caso, existe una teoría subjetiva mantenedora de la acción de enseñanza.

- Profesor 6: Mi formación. A ver, aquí hay que mirar para qué se enseña, se enseña para el futuro, o sea, no se enseña para el presente, digamos, porque el futuro ciudadano es que se va a desempeñar en diversas cosas, en fin, y él tiene 
que llevar una serie de valores y de conocimientos y cosas que le van a servir para su desempeño, entonces, la enseñanza es para el futuro.

\section{DISCUSIÓN}

La formación de profesor es un proceso complejo, que ocurre durante una larga etapa de su vida, resulta de experiencias formales e informales de aprendizaje y que, desde este estudio, se puede caracterizar por medio de cuatro ejes o categorías obtenidas mediante la codificación axial. Cada una de ellas incluye teorías subjetivas que relacionan experiencias de aprendizajes formales y biográficos con la formación docente. La primera de ellas, primeros pasos en la construcción del significado de la profesión docente: formación precoz de la profesión, incluye teorías subjetivas que vinculan experiencias biográficas ocurridas antes de la formación inicial con la formación profesional; la segunda, aprendizaje de la profesión docente durante la formación inicial, aquellas teorías subjetivas que relacionan con la formación las experiencias ocurridas durante ese período; la tercera, el aprendizaje de la profesión durante el ejercicio profesional, considera teorías subjetivas que establecen un vínculo entre las experiencias ocurridas durante el ejercicio profesional y la formación; por fin, orientación a la acción del profesor desde las teorías subjetivas: el sentido de la profesión, de la enseñanza y la propia formación, es una categoría cuyas teorías subjetivas permiten hipotetizar el para qué de la formación docente, el ejercicio de la profesión y la enseñanza.

Planteamos aquí que el conocimiento profesional del profesor se va constituyendo por medio de una serie de experiencias formales e informales de aprendizaje (Torres, 1998). El docente adquiere conocimientos de tipo biográficos, que responden fundamentalmente al cómo enseñar y regulan el comportamiento con un relativo grado de inconsciencia, es decir, un conocimiento en algún nivel implícito (Putnam; Borko, 2000 apud Badía; Monereo, 2004). En ese tipo de conocimiento, una posición importante la tiene la formación precoz de la profesión docente. Tal proceso comienza antes de la formación inicial e implica un aprendizaje de conocimientos fundamentalmente biográficos, adquiridos mientras alumno de enseñanza básica y media, que se asocian fundamentalmente al cómo enseñar y se organizan en la estructura cognitiva con un relativo grado de conciencia. Desde esos hallazgos, los docentes van desarrollando la vocación por la profesión, y a la vez adquieren conocimientos sobre prácticas pedagógicas de tipo académicas, y aprenden a regular la relación profesor-alumno. Este hallazgo nos parece de alta relevancia, dado que, por una parte, implica un aprendizaje de conocimiento profesional que se adquiere siendo alumno(a) por medio de una prologada interacción con su profesor, es decir, que siendo uno estudiante desarrolla ese conocimiento durante cerca de trece años de su vida, diez meses al año, cinco días a la semana y prácticamente ocho horas diarias, situación que no se presenta en ninguna otra profesión.

El segundo elemento que hace pertinente este análisis se sustenta precisamente en la mínima formación pedagógica (didácticas o métodos de enseñanza) de las instituciones de educación superior y su enfoque en la enseñanza de teorías científicas (Duhalde; Cardelli, 2001), situación muy criticada por el docente, lo que conlleva preguntar por el origen del conocimiento pedagógico del profesor. Una 
posible respuesta es que la formación precoz de la profesión docente resulta un insumo importante para la elaboración de teorías subjetivas que regulan las didácticas de enseñanza. Se hace necesario, entonces, que se instalen espacios formativos que permitan el reconocimiento, de parte del alumno de pedagogía, del conocimiento precoz de la profesión, su análisis y relación con las futuras prácticas pedagógicas.

En tal sentido, asumimos la proposición de Schön (1992), Catalán y Castro (2008) de la relevancia del acto reflexivo sobre el propio pensamiento y las prácticas pedagógicas, aunque en este caso, incluyendo un proceso de autoconocimiento respecto a los episodios de la historia vital estudiantil relacionados con lo educativo, además de un análisis crítico que posibilite el reconocimiento, la validación o reconstrucción, no sólo desde lo científico, sino que además desde lo valorativo y lo pragmático. Implica someter a revisión la fuente de conocimiento, la manera como nutre la elaboración de teorías subjetivas durante la formación inicial y el ejercicio de la profesión, y la proximidad que tiene con el aprendizaje de teorías científicas de lo educativo. Lo propuesto consiste en no sólo hacer explicitable el contenido implícito de las teorías subjetivas elaboradas, sino que además, desarrollar activamente un metaconocimiento de las mismas, y, por lo tanto, de lo educativo.

En un período de menor duración, correspondiente a la formación inicial, el futuro docente aprende fundamentalmente conocimientos semánticos del tipo teorías científicas, que regulan qué enseñar, y en menor grado, conocimientos de procedimientos referidos a cómo enseñar, situación que muy criticada por el docente en ejercicio. En esta etapa, el conocimiento de la profesión desde las experiencias biográficas sigue desarrollándose, destacando las experiencias vividas con el grupo de pares y el modelado de profesores, lo que se puede resumir en el currículum oculto de la formación universitaria docente.

El proceso de práctica profesional resulta altamente valorado y posicionado por los profesores como una experiencia de intenso aprendizaje, sobre todo cuando se lleva a cabo en escuelas con índices de vulnerabilidad social importante, dado el reto que imponen. Esto último reviste particular importancia, dado que es posible que el aprendizaje de tales teorías subjetivas se dé en algún nivel implícitamente, es decir, desde la observación de comportamientos de ejercicio profesional de los profesores del centro de práctica, cuyas teorías subjetivas que sustentan sus acciones no son explicitadas; y en menor grado, desde teorías subjetivas de contenido explícito. Dados estos hallazgos, esto cobra sentido si consideramos que los docentes refieren la existencia de un mínimo de espacios formadores formales de la profesión dentro de la escuela, lo que disminuye la posibilidad de análisis y reflexión de lo educativo. Según Rodrigo, Rodríguez y Marrero (1993), lo anterior se corresponde con la génesis social de las teorías implícitas.

Durante el ejercicio de la profesión se inicia una nueva etapa formativa. Corresponde a un período en el que el profesor integra lo que ha aprendido, en gran parte, en teorías subjetivas que representan su posición personal frente a lo educativo. La organización de este conocimiento es compleja y aún se encuentra en discusión. Por ejemplo, Castro (2008) advierte sobre la posibilidad de encontrar teorías subjetivas con algún grado de inconsistencia entre aquellas representadas superficialmente o explícitas respecto de las que se ubican en un nivel más profundo 
(implícitas), atribuyéndole a estas últimas mayor generalidad, arraigo, profundidad y estabilidad (Krause, 2005).

Los hallazgos manifiestan una teoría subjetiva de contenido explícito, de uso recurrente, con contenido valorativo y colectiva, que desvaloriza el tipo de enseñanza recibida durante la formación inicial y la continua, dada la baja aplicabilidad del conocimiento. Junto con esto, se evidencia una gran cantidad de experiencias biográficas que sirven de insumos para la construcción de teorías subjetivas que se encuentran en la base de lo educativo, por lo que podría ser posible que las prácticas pedagógicas se regulen, en algún nivel, tomando por base estas teorías.

Esto se corresponde con los hallazgos que otras investigaciones han demostrado, por ejemplo, respecto a teorías subjetivas simples de docentes sobre enseñanza y aprendizaje (Cuadra, 2009), el bajo estatus teórico de estas teorías planteado por Catalán $(1997,2004)$ o el mínimo impacto de la formación inicial en el ejercicio de la profesión docente (Vezub, 2007). Lo anterior contribuye más a la comprensión cuando se analiza desde lo que aquí planteamos, esto es, que la posición personal representada fundamentalmente por explicaciones o teorías subjetivas del profesor frente a lo educativo se construye intensamente durante ese período, siendo una fuente importante de información las experiencias informales o biográficas de aprendizaje ocurridas antes y durante el ejercicio profesional.

Es posible afirmar la presencia en el profesor de determinados sentidos de lo educativo en función de las características de las teorías subjetiva que se encuentren en la base. En esta investigación, referimos un tipo particular de teorías subjetivas del profesor cuya orientación a la acción permite identificar determinados sentidos en la base de lo que enseña, de su profesión y proceso formativo. Se trata de explicaciones que los docentes presentan cuando reflexionan sobre la función que le otorgan a la profesión, la enseñanza y su formación profesional. Esto es consistente con los hallazgos referidos a los resultados que los profesores desean lograr o piensan que se pueden lograr mediante la enseñanza de valores: resultados posibles y resultados ideales (Castro, 2008; Castro; Cárcamo, 2012). Implica, en algún nivel, un juicio epistemológico y/o valorativo, coherente con las investigaciones de creencias epistemológicas del profesor.

Hipotetizamos que determinados sentidos de lo educativo pueden asociarse a un mejor o peor ejercicio de la profesión. Por ejemplo, y desde los hallazgos encontrados, el ejercicio profesional puede ser impulsado sobre todo porque adquiere significancia a medida que se logra mayor estatus social y mejor ingreso económico, o desde un sentido social de la profesión, básicamente porque adquiere significancia en la posibilidad de contribuir al progreso de la sociedad. Creemos que es necesario profundizar en investigaciones que den cuenta de la orientación a la acción de las teorías subjetivas, a fin de responder a interrogantes no manifestadas desde estos hallazgos, por ejemplo, cuánto impacta el sentido de la profesión, de la enseñanza y la formación en la conducta del profesor, qué tipo de sentidos se relacionan con mejores desempeños profesionales, de qué manera se van configurando esos sentidos o cuáles son las causas de base; y por fin, de qué manera se puede pretender un sentido de esos factores más vinculado con prácticas pedagógicas exitosas.

Como consecuencias metodológicas y prácticas, es prudente que los programas de formación inicial y continua no sólo consideren el sentido que el docente le 
atribuye a lo educativo, sino que además busquen impactarlo por medio de espacios de análisis crítico sobre el para qué de la enseñanza, la formación y la profesión docente. Ello implica llevar el análisis a un nivel epistemológico y valorativo de esos tópicos.

Ubicando la reflexión en las características estructurales de las teorías subjetivas encontradas, desde esos hallazgos se observa un tipo que denominamos central, que se caracteriza por ser ampliamente compartida (colectiva), considerar un juicio valorativo y epistemológico respecto al propio conocimiento, poseer valor explicativo otorgado por el propio sujeto y aplicabilidad. Es una teoría subjetiva que dentro de ese grupo de docentes es ampliamente compartida y validada, en la que destaca un juicio crítico respecto al propio conocimiento profesional, además de una posición valorativa del proceso formativo, denotándose en forma recurrente en sus planteamientos para explicar la formación del profesor.

Una teoría subjetiva central en estos hallazgos refiere a la formación inicial y continua del profesor, y su contenido latente se puede representar de la forma siguiente: hoy día se encuentra una baja calidad en la formación docente, dado que se enseñan fundamentalmente conocimientos teóricos que no se ajustan a la realidad escolar, debido a las características de los formadores de formadores y a las políticas educativas, que no cuidan a la calidad de la enseñanza y facilitan las ganancias con los procesos formativos. Así que queremos reflexionar acerca del influjo en la conducta profesional del profesor, así como en sus procesos de cambio. En primer lugar, se infiere una orientación a la acción que inhibe su inserción en procesos de formación continua o genera bajas expectativas cuando participa de ellos, lo que permitiría comprender la mínima transferencia de lo aprendido al aula o el bajo impacto de la formación inicial en las escuelas (Vezub, 2007) y la simplicidad de sus explicaciones de lo educativo (Catalán, 2004; Cuadra, 2009).

En segundo lugar, respecto a la estructura de ese tipo de teorías subjetivas, parece ser que la complejidad de ese tipo de conocimiento no sólo está dado por la profundidad de la teoría subjetiva (o el nivel implícito que presenta) o el estatus teórico respecto al nivel de organización que alcanza en contrastación con la estructura de las teorías científicas; sino que, además, por el grado en que ésta es compartida por un grupo social (colectiva), su aplicabilidad (o su recurrencia para explicar los hechos), y la metacognición que alcanza en la asunción de un juicio valorativo y epistemológico del propio conocimiento (en este caso, del valor explicativo que el sujeto otorga a la teoría subjetiva y el cuestionamiento valorativo de algo aprendido). Es posible que ese tipo de teorías subjetivas guíen con fuerza la acción del profesor, en este caso, de la manera en cómo aborda su proceso formativo. Dado lo anterior, consideramos pertinente llevar a cabo una indagación y abordaje inicial de ese tipo de conocimiento a la hora de instalar instancias formativas, puesto que podrían condicionar el aprendizaje del docente. En segundo lugar, sugerimos profundizar en la investigación de ese tipo de teorías subjetivas, dado que eso podría poner de manifesto de qué manera los propios sujetos, y no sólo el investigador, le otorgan poder explicativo, valor y complejidad a esas teorías.

Por fin, sólo resta señalar que la discusión desarrollada debe asumirse a modo de hipótesis y para unidades de análisis similares a la estudiada. La pretensión de esta investigación ha sido únicamente contribuir en comprensión respecto a un 
aspecto clave para la eficacia educativa, es decir,la formación docente, pero desde un modelo teórico que ubica el conocimiento del profesor, específicamente las teorías subjetivas, como fundamento de sus prácticas educativas.

\section{Cuadro 2 - Codificación selectiva sobre el proceso de formación docente}

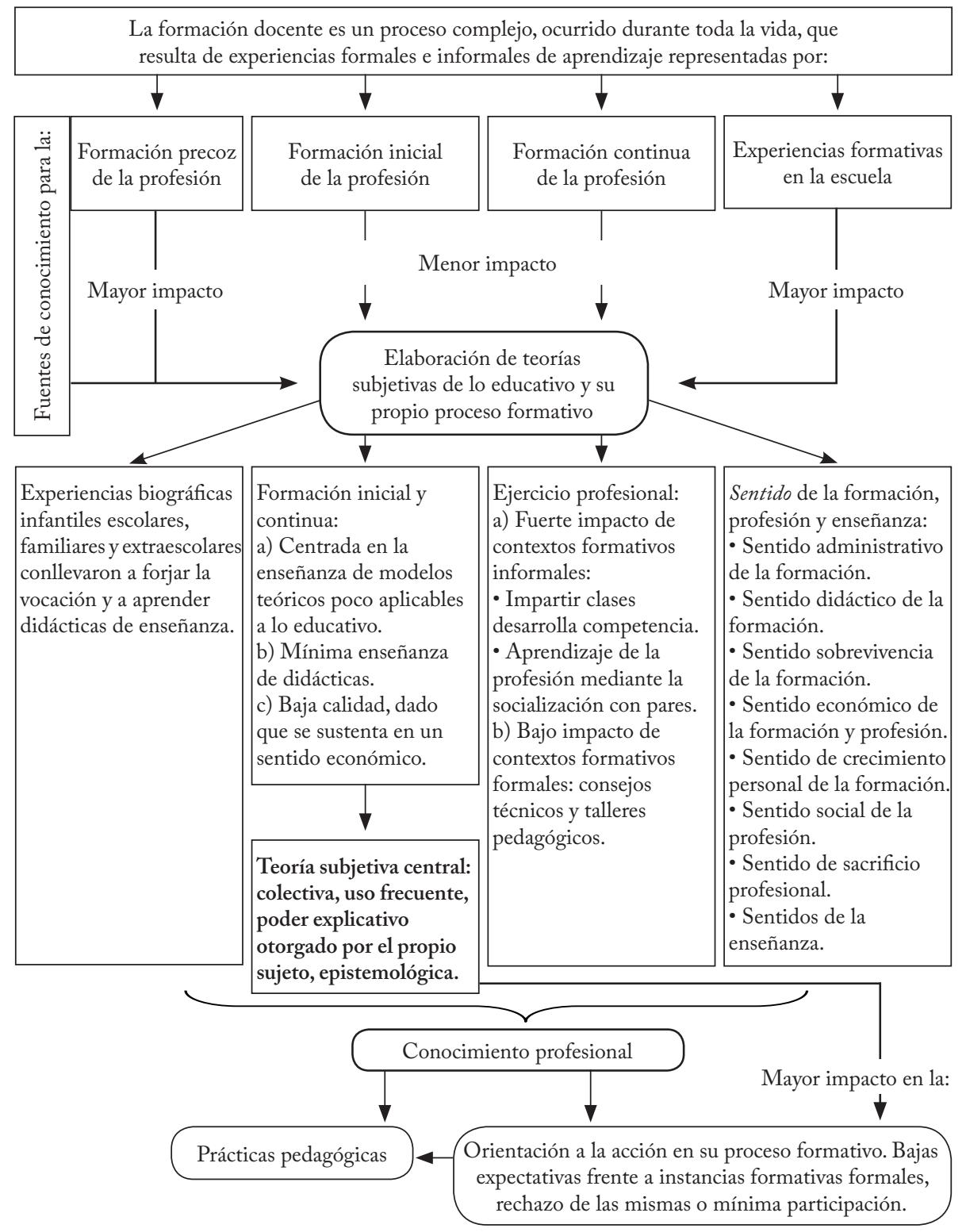

Fuente: Rectángulos que contienen el texto Formación precoz de la profesión, Formación inicial de la profesión, Formación continua de la profesión, Experiencias formativas en la escuela. 


\section{REFERENCIAS}

Arístegui, R. et al. Actos de habla en la conversación terapéutica. Terapia Psicológica, Santiago de Chile: Sociedad Chilena de Psicología Clínica,v.22, n. 2, p.131-143, nov. 2004. Badía, A.; Monereo, C. La construcción de conocimiento profesional docente. Análisis de un curso de formación sobre la enseñanza estratégica. Anuario de Psicología, Barcelona: Universitat de Barcelona; Horsori Editorial v. 35, n. 1, p. 47-70, mar. 2004.

CAstro, P. Cambio de teorías subjetivas de profesores respecto a la enseñanza y el aprendizaje de valores. 2008. 206f. Tesis (Doctorado en Psicología) - Pontificia Universidad Católica de Chile, Facultad de Ciencias Sociales, Santiago de Chile, Chile, 2008.

.; CÁrCamo, R. Cambio de teorías subjetivas de profesores respecto a la educación en valores. Subjetividad y procesos cognitivos, Buenos Aires: Universidad de Ciencias Empresariales y Sociales, v. 16, n. 1, p. 17-42, jun. 2012.

Catalán, J. La profesión del profesor según las teorías subjetivas de los formadores. 1997. 649f. Tesis (Doctorado en Ciencias de la Educación) - Pontificia Universidad Católica de Chile, Facultad de Educación, Santiago de Chile, Chile, 1997.

.Formación inicial de educadoras de párvulos: un estudio de caso desde las teorías subjetivas de formadores y formadoras. Revista Iberoamericana de Educación, Madrid: Organización de Estados Iberoamericanos para la Educación, la Ciencia y la Cultura, v.5, n. 33, p. 1-14, jul. 2004. Disponible en: <http://www.rieoei.org/investigacion7.htm>. Acceso en: 24 nov. 2011.

Análisis de contenido en estudios de teorías subjetivas. In: Congreso DE Metodología de las Ciencias Sociales y de la Salud, 9., 2005, Granada.Anais... Granada: Programa Científico del IX Congreso de Metodología de las Ciencias Sociales y de la Salud, 2005. Disponible en: <http://www.ugr.es/ cmetodo/pdf/comunicaciones/ catalan_ahumada.pdf $>$. Acceso en: 24 nov. 2011.

. Teorías subjetivas. Aspectos teóricos y prácticos. La Serena: Editorial Universidad de La Serena, 2010.

Del pensamiento al conocimiento profesional del profesor. In: (Org.). Psicología Educacional. Proponiendo rumbos, problemáticas y soluciones. La Serena: Editorial Universidad de La Serena, 2011. p. 187-215.

.; CAstro, P. Proyecto DIULS. Reflexión colectiva sistemática en el desempeño docente: un estudio orientado al desarrollo profesional. La Serena: Universidad de La Serena, 2008.

Clark, C.; Peterson, P. Procesos de pensamiento de los docentes. In: Wittrock, M. (Org.). La investigación de la enseñanza. 3. ed. Barcelona: Paidós, 1990. p. 444-543.

CuAdra, D. Teorías subjetivas en docentes de una escuela de bajo rendimiento, sobre la enseñanza y el aprendizaje del alumno. Revista Mexicana de Investigación Educativa, Ciudad de México: Consejo Mexicano de Investigación Educativa,v. 14, n. 42, p. 939-967, sept. 2009.

Davini, M. La formación docente en cuestión: política y pedagogía. Buenos Aires: Paidós, 1995. 
De Lella, C. Modelos y tendencias de la formación docente. In: Seminario Taller sobre Perfil del Docente y Estrategias de Formación, 1., 1999, Lima. Anais... Lima: Organización de Estados Iberoamericanos para la Educación, la Ciencia y la Cultura, 1999. Disponible en: <http://www.oei.es/cayetano.htm>. Acceso: 13 dic. 2012. Duhalde, M.; Cardelli, J. Formación docente en América Latina. Una perspectiva político-pedagógica. Cuadernos de Pedagogía, Madrid: Wolters Kluwer España, n. 308, p. 38-45, dic. 2001.

FLick, U. Introducción a la investigación cualitativa. Madrid: Ediciones Morata, 2004.

Fox, D. Personal theories of teaching. Studies in Higher Education, Nottingham: Society for Research into Higher Education, v. 8, n. 2, p. 151-163. 1983.

Fullan, M. Los nuevos significados del cambio en educación. Barcelona: Octaedro, 2002. Groeben, N.; Scheele, B. Dialog-Konses-Methodik im Forschungsprogramm Subjektive Theorien. Forum Qualitative Sozialforschung, Berlin: Universität Berlin, v. 1, n. 2, artículo 10. 2000. Disponible en: <http://qualitative-research.net/fqs/fqs-d/200inhalt-d.htm>.

Grotjahn, R. The Research Programme Subjective Theories. Studies in Second Language Acquisition, Cambridge: Cambridge University Press, v. 13, p. 187-214, 1991.

Jiménez, A.; Feliciano, L. Pensar el pensamiento del profesorado. Revista española de pedagogía, La Rioja: Universidad Internacional de La Rioja, n. 233, p. 105-122, enero-abr. 2006. Disponible en: <http://revistadepedagogia.org/vol.-lxiv-2006/n\%C2\%BA-233enero-abril-2006/>. Acceso en: 13 enero 2012.

Krause, M. La investigación cualitativa: un campo de posibilidades y desafíos. Revista Temas de Educación, La Serena: Editorial Universidad de La Serena, n. 7, p. 19-39, 1995.

. Psicoterapia y cambio. Una mirada desde la subjetividad. Santiago de Chile: Ediciones Universidad Católica de Chile, 2005.

Manzi, J.; González, R.; Sun, Y. La evaluación docente en Chile. Santiago de Chile: MIDE UC, Centro de Medición, Pontificia Universidad Católica de Chile, 2011.

Marcelo, C. El pensamiento del profesor. Barcelona: CEAC, 1987.

Introducción a la formación del profesorado. Teoría y Métodos. Sevilla: Editorial Universidad de Sevilla, 1989.

. Las tecnologías para la innovación y la práctica docente. Revista Brasileira de Educação, Rio de Janeiro: ANPEd; Campinas: Autores Associados, v. 18, n. 52, p. 25-47, mar. 2013. Disponible en: <http://www.scielo.br/pdf/rbedu/v18n52/03.pdf $>$. Acceso en: 31 mar. 2013.

Martínez, M. M. et al. ¿Qué pensamiento curricular y profesional tienen los futuros profesores de ciencias de secundaria? Enseñanza de las Ciencias: Revista de Investigación y Experiencias Didácticas, Barcelona: Universidad Autónoma de Barcelona: Institut de Ciències de l'Educació, ICE, v. 19, n. 1, p. 67-87, 2001.

Ministerio de Educación. La educación chilena en el cambio de siglo: políticas, resultados y desafíos. Informe Nacional de Chile, Santiago de Chile: Oficina Internacional de Educación, UNESCO, 2004. 
Moral, C. Formación para la profesión docente. Revista Interuniversitaria de Formación del Profesorado, Zaragoza: Asociación Universitaria de Formación del Profesorado, n. 37, p. 171-186, abr. 2000.

Putnam, R.; Bоrко, H. What do new views of knowledge and thinking have to say about research on teacher learning? Educational Researcher, Washington, DC: American Educational Research Association, v. 29, n. 1, p. 4-15.Jan./Feb. 2000.

Raczynski, D.; Muñoz, G. Reforma educacional chilena: el difícil equilibrio entre la macro y la micro-política. REICE: Revista Iberoamericana sobre Calidad, Eficacia y Cambio en Educación, Madrid: Red Iberoamericana de Investigación sobre Cambio y Eficacia Escolar, v. 5, n. 3, p. 40-83, 2007. Disponible en: <http://dialnet.unirioja.es/ servlet/articulo?codigo=2354541>. Acceso en: 23 jun. 2012.

Rodríguez, A. Problemas, desafíos y mitos en la formación docente. Perfiles Educativos, Ciudad de México: Instituto de Investigaciones sobre la Universidad y la Educación, n. 63, p. 3-7, enero-mar. 1994.

Rodrigo, M.J.; Rodríguez, A.; Marrero, J. Las teorías implícitas. Una aproximación al conocimiento cotidiano. Madrid: Visor Distribuciones, 1993.

Ruiz, J. Metodología de la investigación cualitativa. 3. ed. Bilbao: Universidad de Deusto, 2003.

Sснӧn, D. La formación de profesionales reflexivos. Barcelona: Paidós, 1992.

Shulman, L. Those who understand: knowledge growth in teaching. Educational Researcher, Washington: American Educational Research Association, v. 15, n. 2, p. 4-14, feb. 1986.

Strauss, A.; Corbin, J. Bases de la investigación cualitativa. Técnicas y procedimientos para desarrollar la teoría fundamentada. 1. ed. Antioquia: Editorial Universidad de Antioquia, 2002.

TAYLOR, S.; BogdAn, R. Introducción a los métodos cualitativos de investigación. Barcelona: Paidós, 1987.

Torres, R. Nuevo papel docente. ¿Qué modelo de formación y para qué modelo educativo? Perfiles Educativos, Ciudad de México: Instituto de Investigaciones sobre la Universidad y la Educación, v. 20, n. 82, p. 6-23, oct./dic. 1998.

Vaillant, D. Formación de docentes en América Latina. Re-inventando el modelo tradicional. Barcelona: Octaedro, 2005.

VEzub, L. La formación y el desarrollo profesional docente frente a los nuevos desafíos de la escolaridad. Profesorado. Revista de Currículum y Formación de Profesorado, Granada: Universidad de Granada, v. 11, n. 1, p. 2-23, 2007.

\section{SOBRE LOS AUTORES}

David Jorge Cuadra Martínez es magíster en psicología por la Universidad de La Serena (Chile). Profesor de la Universidad Santo Tomás (Chile).

E-mail: cuadradavid2@gmail.com 
Jorge René Catalán Ahumada es doctor en ciencias de la educación por la Pontificia Universidad Católica de Chile (Chile). Profesor de la Universidad de La Serena (Chile).

E-mail: jcatalan@userena.cl

Recebido em dezembro de 2013

Aprovado em março de 2015 